\title{
EXPERIENCIA PRELIMINAR CON CINTA DE TERCERA GENERACIÓN EN EL TRATAMIENTO DE LA INCONTINENCIA DE ORINA DE ESFUERZO: MINIARC
}

\author{
Jack Pardo S., Vicente Solà D., Paolo Ricci A.
}

Unidad de Ginecología, Departamento de Obstetricia y Ginecología, Clínica Las Condes.

\section{RESUMEN}

Antecedentes: Durante la última década se han desarrollado numerosas técnicas para tratar quirúrgicamente la incontinencia de orina de esfuerzo (IOE), entre ellas las minicintas o mallas de tercera generación. Objetivos: Determinar la eficacia y seguridad del tratamiento de la IOE, mediante cinta MiniArc. Método: Estudio prospectivo, a corto plazo, de 24 mujeres ingresadas a la Unidad de Uroginecología de Clínica Las Condes, entre abril de 2008 y abril de 2009. Criterio de inclusión: IOE pura, al menos por un año, de carácter permanente y objetivada por urodinamia de monocanal. Todas debían tener hipermobilidad uretral e incontinencia de esfuerzo tipo II o II + III. En caso de prolapso genital, no debía constituir la indicación primaria de cirugía. Criterio de exclusión: Antecedente de cirugía por IOE y/o prolapso genital. Resultados: Tiempo operatorio con media de 8 minutos. Seguimiento preliminar con media de 6 meses. No hubo complicaciones en el intraoperatorio. Hubo un caso de obstrucción uretral en el postoperatorio inmediato. Al tercer 3 meses se registró un caso de urgencia de novo leve, con solución espontánea al mes de seguimiento. A los 30 días se registró cura en $23(95,8 \%)$, mejoría en $1(4,2 \%)$ y no hubo casos de falla. Conclusiones: El sistema MiniArc, en pacientes seleccionadas, es una técnica segura y eficaz en el tratamiento de la IOE. La incorporación de nuevos pacientes y el seguimiento a largo plazo, permitirá determinar la persistencia de estos buenos resultados.

\section{PALABRAS CLAVE: Incontinencia de orina de esfuerzo, malla sub-medio uretral, tratamiento quirúrgico}

\section{SUMMARY}

Background: During the last decade we observed the development of numerous surgical techniques to treat stress urinary incontinence (SUI). One of these techniques is the MiniArc. Objective: To determine the efficacy and safety of the surgical treatment of SUI by MiniArc. Method: Prospective study of short follow-up, of 24 women admitted in the Urogynecology of Clínica Las Condes, between April 2008 and April 2009. Inclusion criteria: SUI at least for one year and permanent, objectified through a mono channel urodynamic. All women should have urethral hyper mobility and urinary incontinence type II or II + III. In cases with genital prolapse, it should not be the primary indication for surgery. Exclusion criteria: Antecedent of surgery for SUI and/or for genital prolapse. Results: The surgical time media of 8 minutes. The preliminary follow-up was between 1 and 13 months, media 6 months. There were no complications during the MiniArc application. In the immediate postoperative period there was a case of urethral obstruction. During the third month, there was a case of mild novo urge incontinence, which was resolved spontaneously after one month. At 30 days after surgery 
in $23(95.8 \%)$ cure was registered, improvement in $1(4.2 \%)$ and cases of failure not were observed. Conclusions: In selected patients, MiniArc is safe and effective in the treatment of SUI. The incorporation of new patients to these protocols and long term follow-up will determine the persistence of these good results.

\section{KEY WORDS: Stress urinary incontinence, sub-mid urethra mesh, surgical treatment}

\section{INTRODUCCIÓN}

Durante los últimos años hemos asistido a un aumento de las técnicas quirúrgicas para la incontinencia de orina de esfuerzo. Hace más de una década que se presentó el primer tratamiento quirúrgico con una malla sub-medio uretral, el clásico TVT $(1,2)$. Este nuevo concepto de una malla libre de tensión, revolucionó todo lo realizado hasta ese momento, convirtiéndose en la base de las nuevas técnicas quirúrgicas con cintas, que se desarrollarían más tarde. Sin embargo, se trata de una malla que pasa por el espacio retropúbico, por lo que no está libre de complicaciones, tales como la perforación de vísceras abdomino-pélvicas, vasos sanguíneos y la vejiga $(3,4)$. Por otro lado, debemos considerar que esta técnica quirúrgica requiere de la utilización de una cistoscopia de revisión, por lo que si no se dispone, no debiera realizarse. Con el objetivo de simplificar esta técnica quirúrgica, se presenta en 2001 la primera cinta sub-medio uretral con salida por el agujero obturador $(5,6)$. De esta forma se evita el paso por el espacio retropúbico, alejándose de la vejiga, por lo que no requiere realizar cistoscopia de revisión de rutina (7). Cuando se pensaba que era imposible una mayor simplificación de estos procedimientos (8), durante el 2006, se presenta una nueva técnica, el TVT-Secur (9). Así nace la tercera generación de mallas sub-medio uretrales, para el tratamiento quirúrgico de la incontinencia de orina de esfuerzo. Otro representante de estas cintas, llamadas también, mini-cintas, debido a su pequeño tamaño, es el MiniArc (10). La principal diferencia entre ambas cintas, es que en el TVTSecur, la malla se fija al hueso púbico, mientras que en el MiniArc, lo hace al agujero obturador (11).

El objetivo de esta comunicación es presentar nuestra experiencia preliminar con los primeros casos de pacientes con tratamiento quirúrgico de la incontinencia de orina de esfuerzo, por medio de la malla de tercera generación MiniArc.

\section{PACIENTES Y MÉTODO}

Protocolo de estudio: Seguimiento prospectivo, para observar los resultados inmediatos sobre la incontinencia de orina de esfuerzo, de 24 mujeres ingresadas a la Unidad de Uroginecología y Cirugía
Vaginal de Clínica Las Condes, para corrección quirúrgica por medio del nuevo sistema de cinta submedio uretral de tercera generación, MiniArc (AMS, Minnetonka, MN, Estados Unidos), entre abril del 2008 y abril 2009.

Criterio de inclusión: Debían presentar incontinencia de orina de esfuerzo pura, al menos por un año y de carácter permanente, con diagnóstico clínico y demostrado por medio de urodinamia monocanal, con medición de la presión de retro-resistencia uretral y cistometrograma $(12,13)$. Todas debían tener hipermobilidad uretral, confirmada por medio de un Q-Tip test e incontinencia de esfuerzo tipo II o II + III de la clasificación de McGuire y cols (14). En caso de presentar prolapso genital, no debía constituir la indicación primaria de la cirugía.

Criterio de exclusión: Antecedente de cirugía para incontinencia de orina de esfuerzo y/o prolapso genital.

Características del grupo de pacientes ingresadas al estudio: La edad media del grupo en estudio fluctuó entre los 39 y 74 años, con una media de 49 años. La paridad fluctuó entre 2 y 5 , con una media de 3. Presentaban incontinencia de orina de esfuerzo tipo II, 20 (83,3\%) mujeres y $4(16,7 \%)$ tipo II+III. Se realizó cirugía asociada en 11 pacientes, correspondiendo a 4 casos de plastía de labios menores por asimetría y/o hipertrofia, 3 esterilizaciones tubarias laparoscópicas y 4 con perineoplastía (una de ellas además con esfinteroplastía anal) (Tabla I).

Tabla I

\section{CARACTERÍSTICAS DE LAS PACIENTES INGRESADAS AL ESTUDIO}

\begin{tabular}{lrc}
\hline Características de las pacientes & Rango & Media \\
\hline Edad (años) & $39-74$ & 49 \\
IMC & $25-32$ & 28 \\
Paridad (hijos) & $2-53$ &
\end{tabular}

Incontinencia por urodinamia

Tipo I: n (\%)

$20(83,3)$

Tipo II + III: n (\%)

$4(16,7)$

Tiempo operatorio (minutos)

$4-11$

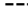

Seguimiento (meses)

$1-13$ 
Descripción del dispositivo MiniArc: Dispositivo compuesto por una aguja de inserción y malla. La misma aguja se utiliza para la inserción de la malla a ambos lados. Está formada por un mango y la aguja propiamente tal, en cuyo extremo se coloca la cinta. Esta es de polipropileno, macroporo, monofilamento, tipo I (15). Posee en sus extremos puntas en forma de "espina de pescado", que permiten que quede inserta en la membrana obturadora, sin posibilidad de retroceso en su posición. La malla en su porción media posee una zona de marca, para identificar la posición sub-medio uretral (Figura 1).

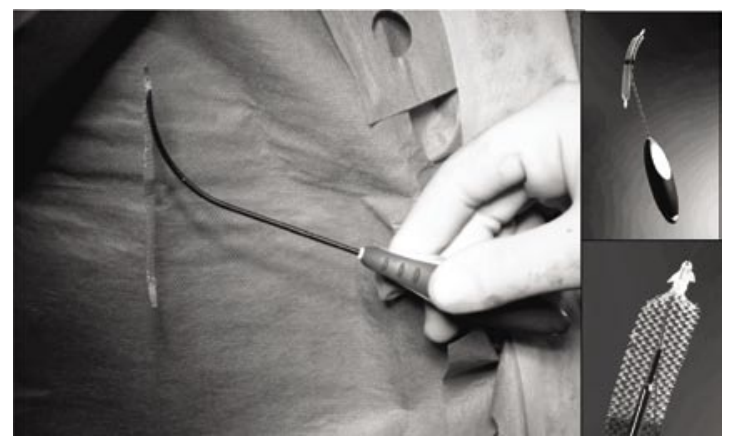

Figura 1. Aguja con la cinta de MiniArc. En el recuadro se observa el detalle de la cinta.

Técnica quirúrgica específica. En posición de litotomía y anestesia espinal, se procede a los siguientes pasos:

Paso 1. Incisión en la mucosa vaginal, porción submedio uretral, a un centímetro del meato urinario, de $1,3 \mathrm{~cm}$ aproximadamente.

Paso 2. Disección bilateral, en 45 grados, de 1 a 1,5 $\mathrm{cm}$, en dirección hacia la porción interior de la rama inferior del hueso púbico (Figuras 2).

Paso 3. Inserción de la malla por medio de la aguja, quedando inserta en la membrana obturadora (Figuras 3,4 y 5 ).

Paso 4. Se retira la aguja, quedando la malla inserta, y se repite lo mismo al lado contra lateral. Cierre de la mucosa vaginal con punto corrido de monocril $3 / 0$

La paciente se levanta y deambula a las 4 horas, con alta hospitalaria luego de verificar 2 micciones espontáneas. Control ambulatorio a los 7 y 30 días, con indicación de control anual para verificar la mantención de los resultados y la aparición de posibles complicaciones durante el postoperatorio tardío. En el control de los 30 días se preguntó por el grado de satisfacción y los resultados obtenidos. Se clasificó el resultado de la cirugía para la incontinencia de orina de esfuerzo, según el número de episodios de pérdida de orina registrados por la paciente, durante el período de observación. Se consideró cura a la ausencia de incontinencia, mejoría a la presencia de episodios menos que una vez cada dos semanas y falla a la presencia de episodios más de una vez por semana (16).

\section{RESULTADOS}

El tiempo operatorio fluctuó entre 4 y 11 minutos, con una media de 8 minutos. El período de observación al cierre del presente informe preliminar, fue entre 1 y 13 meses, con una media de 6 meses.

No se registraron complicaciones debido a la inserción del MiniArc, durante el período intraoperatorio. En el postoperatorio inmediato (primeras 24 horas) se registró un caso de obstrucción uretral. Se trataba de una mujer de 59 años, a quien se le asoció una colpoperineoplastía y una esfinteroplastía

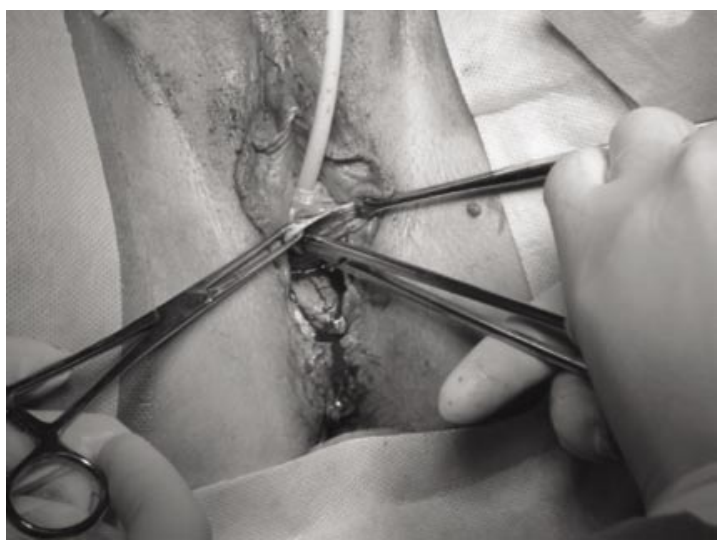

Figura 2. Disección bilateral, en 45 grados, de 1 a 1,5 centímetros, en dirección hacia la porción interior de la rama inferior del hueso púbico.

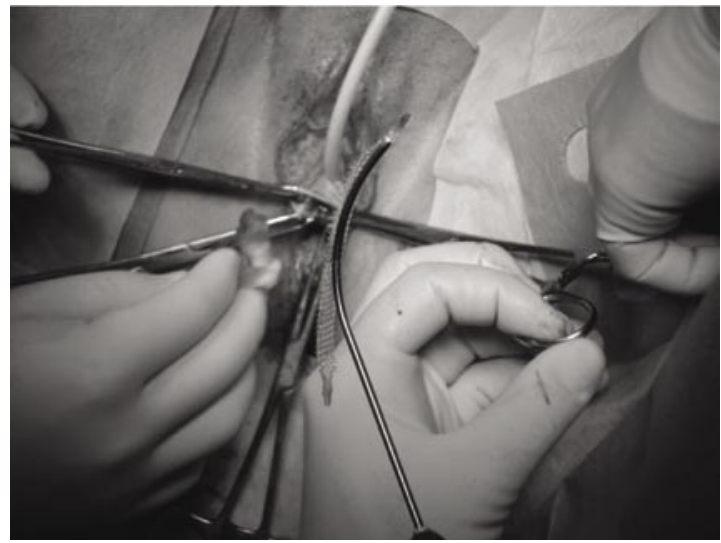

Figura 3. Aguja con la malla, lista para su introducción. 
anal (realizada en conjunto con coloproctólogos); a las 24 horas en pabellón, se bajó la malla por medio de tracción y ajuste con tijera de Metzembaum entre esta y la uretra, lográndose continencia normal.

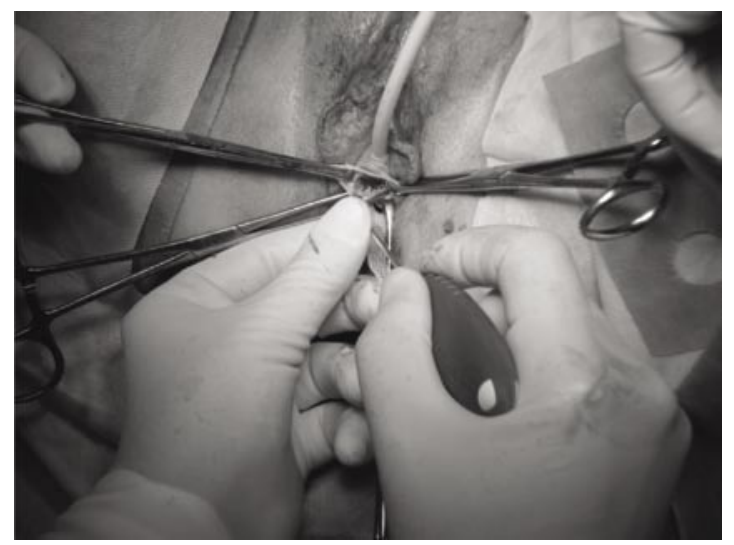

Figura 4. Detalle de la introducción al lado izquierdo de la malla de MiniArc.

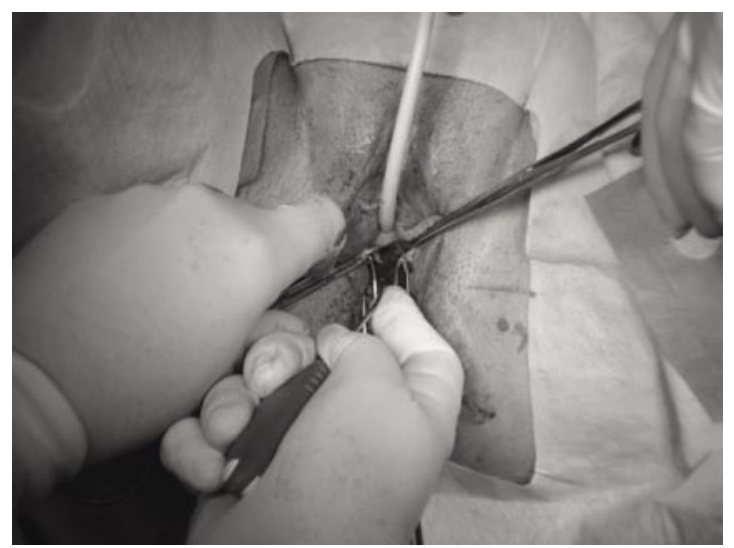

Figura 5. Detalle de la introducción al lado derecho de la malla de MiniArc.

Se registró una complicación durante el tiempo intraoperatorio, debido a cirugía agregada. Se trataba de una mujer de 47 años, en la que se asoció una malla Perigee, debido a prolapso genital, perforándose la pared lateral de la vejiga y observada por cistoscopia; se retiró y reubicó la aguja, quedando con sonda Foley por $48 \mathrm{hrs}$, sin interferir en el resultado de la cirugía por incontinencia.

En el postoperatorio tardío (3 meses), se registró un caso de urgencia de novo leve, que se solucionó espontáneamente al mes de evolución. A los 30 días del postoperatorio, se observó cura en 23 pacientes $(95,8 \%)$, mejoría en $1(4,2 \%)$, y no hubo casos de falla. Al preguntar por el grado de satisfacción a los 30 días, todas expresaron estar conformes con la cirugía.

\section{DISCUSIÓN}

El estándar de oro quirúrgico para la incontinencia de orina de esfuerzo, es considerado hoy el TVT. La cirugía de Burch ha sido desplazada por esta técnica, con la cual ya se han publicado los once años de seguimiento prospectivo (17), demostrándose un $90 \%$ de cura.

Son pocos los trabajos que existen con MiniArc, debido a que se trata de un instrumento muy nuevo. Actualmente sólo existen tres trabajos en revistas indexadas en Medline. En una de estas publicaciones se analizaron 72 casos, evaluados a los dos meses del postoperatorio, demostrándose un $75,7 \%$ de cura de la incontinencia de esfuerzo (79,6\% para IOE pura, $66,6 \%$ para IOE con deficiencia intrínseca de esfínter y $61,5 \%$ para incontinencia mixta), y en un $2,9 \%$ se constató falla (10).

Otra publicación, analizó 97 mujeres con incontinencia de esfuerzo o mixta. El $61,7 \%$ se sometía a cirugía por recurrencia. Durante el tiempo intraoperatorio se presentó un caso de hematoma y un caso de perforación de vejiga. En esta serie llama la atención que el 36,8\% registró aparición de urgencia de novo. Los resultados a corto plazo, demostraron que a las 6 semanas después de la cirugía, la prueba de la tos fue negativa en $79(83,1 \%)$ mujeres. En $74(77,8 \%)$ continuaba negativa a los 12 meses. Al año la calidad de vida mejoró significativamente en $65(69,1 \%, p<0,001)$, con disminución del número de apósitos de 2,2 a 0,6 (p<0,001) (18).

En la tercera publicación, se compararon las dos técnicas de mini-cintas de tercera generación, el TVT-Secur y el MiniArc (11), en base a las complicaciones y resultados a corto plazo. Se registró sólo un caso de perforación vesical con TVT-Secur. Respecto a la cura, fue en un $80,4 \%$ en el grupo de TVT-Secur y de 90,2\% para MiniArc, sin encontrarse diferencias significativas para ambos grupos.

Algunos trabajos expuestos en congresos de la especialidad, durante el 2007 y 2008, han demostrado porcentajes de cura entre un 86 y $98 \%$ (19). Se trata de trabajos de resultados medidos a corto plazo, como es habitual cuando es introducida en forma reciente una nueva técnica, y con diversidad de métodos para objetivar la cura. Algunos la miden en base a la prueba de la tos, "pad test o apósitos", o cuestionarios de calidad de vida.

Otro punto importante a discutir es la utilización de estas cintas en pacientes con valsalva crónico. Se trata de cintas más cortas, que teóricamente podrían correrse al verse sometidas a presiones 
mayores de lo habitual, antes de alcanzar su biointegración con el uroligamento que pretenden reforzar (20).

Sin embargo, no debemos olvidar que lo importante para reparar o reforzar los uroligamentos, no es la porción distal de las mallas (extremos), y sí lo es la porción que queda en la parte sub-medio uretral. Además, todas las cintas, son dejadas como mallas libres de tensión, tal como es condición desde la instalación del primer TVT retropúbico clásico.

En general, debemos reconocer que en los casos de mini-cintas, y en base a la experiencia de nuestro grupo de trabajo, las dejamos con un poco más de tensión que los TVT o los TOT y TVT-O. Sin embargo, no olvidamos que se trata de cintas libre de tensión.

En el caso de la cinta TVT-secur, primero fue sometida a estudios en animales. Es así como se revisó la integración a los tejidos en ovejas, y se midió la fuerza de tensión que lograban bajo la uretra. La fuerza de fijación entregada por este sistema, es inicialmente equivalente a la otorgada por el TVT y aumenta en el tiempo. Además se demostró que se requiere una fuerza mayor a $5 \mathrm{~N}(500 \mathrm{~g})$, para lograr deformar la cinta. Esto equivale a una fuerza 10 veces mayor a la tensión media soportada por la fascia en situación normal (21).

En una experiencia recientemente publicada por nuestro grupo de trabajo, en 110 pacientes con TVT-Secur, con una media de seguimiento de 8 meses (seguimiento máximo de 19 meses), se registró un $95,5 \%$ de cura, 3,6\% de mejoría y $0,9 \%$ de falla. Además un $1,8 \%$ presentó urgencia miccional de novo. Durante la cirugía se registraron 2 casos de perforación vesical. En el postoperatorio inmediato se reajustaron 2 cintas por obstrucción (20).

Aún cuando ambos sistemas de mini-cintas de tercera generación, son sistemas nuevos, utilizan una cinta que no es nueva, siendo la misma utilizada por todos los sistemas clásicos. Se trata de una malla de polipropileno, macroporo, monofilamento, utilizada por el TVT clásico, y por el TOT y TVT-O, de eficacia comprobada. Esta es una malla clasificada como Tipo I (22). Por otro lado, el MiniArc utiliza como punto de anclaje, el mismo sitio de las técnicas obturadoras, también de eficacia comprobada.

Muchos grupos de trabajo, se han opuesto a trabajar con las mini-cintas, esperando que aparezcan trabajos con grandes series bajo estas técnicas. Sin embargo, no debemos olvidar lo expuesto anteriormente, no son cintas nuevas, sólo son más pequeñas, buscando una menor invasión aún, respecto a las técnicas antecesoras. No debemos sentirnos presionados a utilizar nuevas técnicas, por parte de esta industria emergente y las pacientes, que cada día llegan más informadas a nuestras consultas, exigiendo lo más moderno para su tratamiento. Actualmente se está desarrollando un protocolo de trabajo para comparar resultados entre TVT y MiniArc (23).

La existencia de múltiples tratamientos quirúrgicos para la incontinencia, sólo demuestra lo complejo que puede ser este tema. La tolerancia a la diversidad de opiniones debe ser una de las guías para continuar en la investigación y comprensión que a diario realizamos. Todo cuanto logremos avanzar, va en directo beneficio de nuestras pacientes.

\section{CONCLUSIONES}

De acuerdo a nuestra experiencia inicial y en pacientes seleccionadas, se trata de una técnica factible y segura en el tratamiento de la incontinencia de orina de esfuerzo. No debemos olvidar que sólo la incorporación de nuevos pacientes a estos protocolos y el seguimiento a largo plazo, permitirá determinar la persistencia de estos buenos resultados.

\section{BIBLIOGRAFÍA}

1. Nilsson SG. The tensionfree vaginal tape procedure (TVT) for treatment of female urinary incontinence. A minimal invasive surgical procedure. Acta Obstet Gynecol Scand Suppl1998;168:34-7.

2. Ulmsten $U$, Falconer $C$, Johnson $P$, Jomaa $M$, Lannér L, Nilsson CG, et al. A multicenter study of tensionfree vaginal tape (TVT) for surgical treatment of stress urinary incontinence. Int Urogynecol J Pelvic Floor Dysfunct 1998;9(4):210-3.

3. Kuuva N, Nilsson CG. A national wide analysis of complications associated with the tension-free vaginal tape (TVT) procedure. Acta Obstet Gynecol Scand 2002;81(1):72-7.

4. Niemczyk P, Klutke JJ, Carlin BI, Klutke CG. United States experience with tension-free vaginal tape procedure for urinary stress incontinence: assessment of safety and tolerability. T Tech Urol2001;7(4):261-5.

5. Delorme E. Transobturator urethral suspension: miniinvasive procedure in the treatment of stress urinary incontinence in women. Prog Urol 2001;11(6):130613.

6. Delorme E, Droupy S, De Tayrac R, Delmas V. Transobturator tape (uratape). A new minimally invasive method in the treatment of urinary incontinence in women. Prog Urol 2003;13(4):656-9.

7. Pardo Schanz J, Ricci Arriola P, Solà Dalenz V, Tacla Fernández X. Trans-obturator-tape (TOT) for the surgical repair of stress urinary incontinence: our experience. Arch Esp Urol 2006;59(3):225-32. 
8. Solà Dalenz V, Ricci Arriola P, Pardo Schanz J. Stress urinary incontinence surgical correction with third generation sub-mid-urethra sling: TVT-Secur. Actas Urol Esp 2008;32(5):522-9.

9. Martan A, Masata J, Svabik K. TVT SECUR Systemtension-free support of the urethra in women suffering from stress urinary incontinence: technique and initial experience. Ceska Gynecol 2007;72(1):42-9.

10. Debodinance P, Delporte P. MiniArc: preliminary prospective study on 72 cases. J Gynecol Obstet Biol Reprod (Paris) 2009;38(2):144-8.

11. Jiménez Calvo J, Hualde Alfaro A, Raigoso Ortega O, Cebrian Lostal JL, Álvarez Bandres S, Jiménez Parra $\mathrm{J}$, et al. Our experience with mini tapes (TVT Secur and MiniArc) in the surgery for stress urinary incontinence. Actas Urol Esp 2008;32(10):1013-8.

12. Solà V, Pardo J, Ricci P, Cohen D. Nuevo sistema de medición urodinámica en la evaluación de la incontinencia urinaria femenina. Experiencia preliminar con urodinamia monocanal con medición de presión de retro-resistencia uretral. Rev Chil Obstet Ginecol 2008;73(1):85-90.

13. Ricci Arriola P, Solà Dalenz V, Pardo Schanz J. Study of female urinary incontinence with single cannel urodynamics: comparison of the symptoms on admission. Analysis of 590 females. Arch Esp Urol 2009;62(2):115-23.

14. McGuire EJ, Fitzpatrick CC, Wan J, Bloom D, Sanvordenker J, Ritchey M, et al. Clinical assessment of urethral sphincter function. J Urol 1993;150(5 Pt 1):14524.
15. Solà V, Pardo J, Ricci P, Guiloff E. Mallas protésicas en el tratamiento quirúrgico del prolapso genital en la mujer. Rev Med Clin Condes. 2005;16(3):168-79.

16. Blaivas JG, Jacobs BZ. Pubovaginal fascial sling for the treatment of complicated stress urinary incontinence. J Urol 1991;145(6):1214-8.

17. Nilsson CG, Palva K, Rezapour M, Falconer C. Eleven years prospective follow-up of the tension-free vaginal tape procedure for treatment of stress urinary incontinence. Int Urogynecol J Pelvic Flor Dysfunct 2008;19(8):1043-7.

18. Gauruder-Burmester A, Poken G. The MiniArc sling in the treatment of female stress urinary incontinence. Int Braz J Urol 2009;35(3):334-41.

19. MiniArc. Clinical Data. Hallado en: http://www.amsminiarc.com/pdf/MiniArc_CinicalSummary_Lisa.pdf

20. Solà Dalenz V, Ricci Arriola P, Pardo Schanz J. Third generation sub-mid urethral mesh: Experience with 110 TVT-Secur. Arch Esp Urol 2009;62(5):376-88.

21. Rezapour M, Novara G, Meier PA, Holste J, Landgrebe S, Artibani W. A 3-month preclinical trial to assess the performance of a new TVT-like mesh (TVTx) in a sheep model. Int Urogynecol J Pelvic Floor Dysfunct 2007;18(2):183-7.

22. Roth CC, Holley TD, Winters JC. Synthetic sling: which material, which approach. Curr Opin Urol 2006;16(4):234-9.

23. Nacional Institute of Health. Tension Free Vaginal Tape (TVT) versus the Miniarc Sling. Hallado en: http://clinicaltrials.gov/ct2/show/NCT00843908 